\title{
THE
}

1988

\section{Comparison of dynamic height measurements from an inverted echo sounder and an island tide gauge in the central Pacific}

\author{
Stephen M. Chiswell \\ University of Rhode Island \\ Mark Wimbush \\ University of Rhode Island, mwimbush@uri.edu \\ Roger Lukas
}

Follow this and additional works at: https://digitalcommons.uri.edu/gsofacpubs

Terms of Use

All rights reserved under copyright.

\section{Citation/Publisher Attribution}

Chiswell, S. M., Wimbush, M., and Lukas, R. ( 1988), Comparison of dynamic height measurements from an inverted echo sounder and an island tide gauge in the central Pacific, J. Geophys. Res., 93( C3), 22772283, doi: $10.1029 / J C 093 i C 03 p 02277$.

Available at: https://doi.org/10.1029/JC093iC03p02277

This Article is brought to you for free and open access by the Graduate School of Oceanography at DigitalCommons@URI. It has been accepted for inclusion in Graduate School of Oceanography Faculty Publications by an authorized administrator of DigitalCommons@URI. For more information, please contact digitalcommons-group@uri.edu. 


\title{
Comparison of Dynamic Height Measurements From an Inverted Echo Sounder and an Island Tide Gauge in the Central Pacific
}

\author{
Stephen M. Chiswell ${ }^{1}$ and Mark Wimbush \\ Graduate School of Oceanography, University of Rhode Island, Narragansett
}

ROGER LUKAS

Joint Institute for Marine and Atmospheric Research, University of Hawaii, Honolulu

\begin{abstract}
An inverted echo sounder (IES) and deep pressure sensor were deployed within $70 \mathrm{~km}$ of a shallow pressure sensor at Palmyra Island $\left(6^{\circ} \mathrm{N}, 162^{\circ} \mathrm{W}\right)$ in the central Pacific. These instruments provided yearlong records of acoustic travel time, deep pressure and sea level. Two independent time series of dynamic height are derived from travel time and sea surface elevation, respectively. The spectra of these tume series are similar, and at the spectral peaks the coherence between them exceeds $99.9 \%$ confidence levels, indicating that travel time can be used to record dynamic height fluctuations. This investigation provides a frequency dependent calibration for the IES in this region. At the energetic low frequencies (periods $\sim 1$ month), this calibration agrees with a calibration by the standard method using conductivity-temperature-depth (CTD) casts. At higher frequencies (periods of $\sim 3$ days), using the CTDderived calıbration may underestımate the amplitude of some processes by as much as $30 \%$.
\end{abstract}

\section{INTRODUCTION}

From mid-February 1985 to mid-February 1986, as part of the Tropical Ocean and Global Atmosphere (TOGA) program, three inverted echo sounders (IES) [Chaplin and Watts, 1984] were deployed near $6^{\circ}, 8^{\circ}$, and $10^{\circ} \mathrm{N}$ along $162.5^{\circ} \mathrm{W}$ in the central Pacific, as a northward extension of a Line Islands array of near-surface pressure recorders (Figure 1). The southernmost IES with temperature and deep pressure sensors was moored at $5^{\circ} 55^{\prime} \mathrm{N}, 162^{\circ} 43^{\prime} \mathrm{W}$ (4324-m depth), $70 \mathrm{~km}$ west of the northernmost shallow pressure gauge at $5^{\circ} 53^{\prime} \mathrm{N}$, $162^{\circ} 05^{\prime} \mathrm{W}$ (4-m depth), in the lagoon at Palmyra Island.

The IES measures the round trip acoustic travel time from the seafloor to the sea surface. Because the speed of sound varies with temperature and salinity, travel time has been used as a measure of various baroclinic parameters, in particular dynamic height. Previous studies using IESs have linked travel time with dynamic height using the qualitative arguments of Watts and Rossby [1977] and the linear empirical calibrations found, for example, in the Gulf Stream [Watts and Johns, 1982] and in the eastern and central equatorial Pacific [Miller et al., 1985; Chiswell et al., 1986].

These empirical calibrations are usually made by regressing dynamic height with synthesized travel time, both computed from a regional set of conductivity-temperature-depth (CTD) casts, which are not necessarily coincident with an actual IES deployment. The regressions often have a high correlation [e.g., Watts and Rossby, 1977], and the slope of the least squares fit is used as a calibration for the IES. These slopes, however, are controlled mainly by the large-amplitude signals, which generally occur at low frequencies. There is no assurance that the same calibration applies to less energetic, generally higher-frequency signals; and from a set of CTD casts it is

\footnotetext{
${ }^{1}$ Now at Joint Institute for Marine and Atmospheric Research, University of Hawaii, Honolulu.

Copyright 1988 by the American Geophysical Union.

Paper number $7 \mathrm{C} 0901$.

0148-0227/88/007C-0901\$05.00
}

not possible to study these high-frequency signals, since they are aliased in the infrequent and irregular sampling.

The temporally coincident and nearly spatially coincident travel-time, deep-pressure, and shallow-pressure time series made near $6^{\circ} \mathrm{N}$ provide an opportunity to compare dynamic height derived from travel time with that derived independently from sea surface elevation. This paper presents such a comparison to determine how well the IES measures dynamic height at periods from 2 days to 4 months. The 1 -year time series of measurements allows us to calibrate the IES as a function of frequency, and we investigate how this calibration compares with one obtained directly from a set of CTD casts.

In the next section we briefly review the arguments relating travel time to dynamic height. Then in section 3 we present a calibration made from CTD casts taken as part of the North Pacific Experiment (NORPAX) Hawaii-to-Tahiti Shuttle. Section 4 develops a model for the interrelationship of travel time, deep pressure, and shallow pressure. The comparison between IES-derived and sea level-derived dynamic height is given in section 5. Section 6 presents the conclusions.

\section{TRAVEl TIME AND ITS INTERPRETATION}

The IES is moored near the ocean bottom and regularly transmits and detects sound pulses to measure the round trip acoustic travel time, $\tau$, from the seafloor to the sea surface. Watts and Rossby [1977] note that since the speed of sound, $c$, varies approximately linearly with density $\rho$, the integral for $\tau$,

$$
\tau=2 \int_{-\eta}^{z_{2}} 1 / c d z
$$

is similar to that for dynamic height $D$,

$$
D_{p_{1} / p_{2}}=10^{3} \int_{p_{1}}^{p_{2}} 1 / \rho d p
$$

where $\eta=$ sea level, $z=$ depth (positive downward), and $p=$ pressure. SI units are used here (depth in meters, density in kilograms per cubic meter, etc.), except for pressure and dynamic height, which are in decibars and dynamic meters (1 dyn $\mathrm{m}=10 \mathrm{~m}^{2} \mathrm{~s}^{-2}$ ), respectively. Watts and Rossby [1977] 


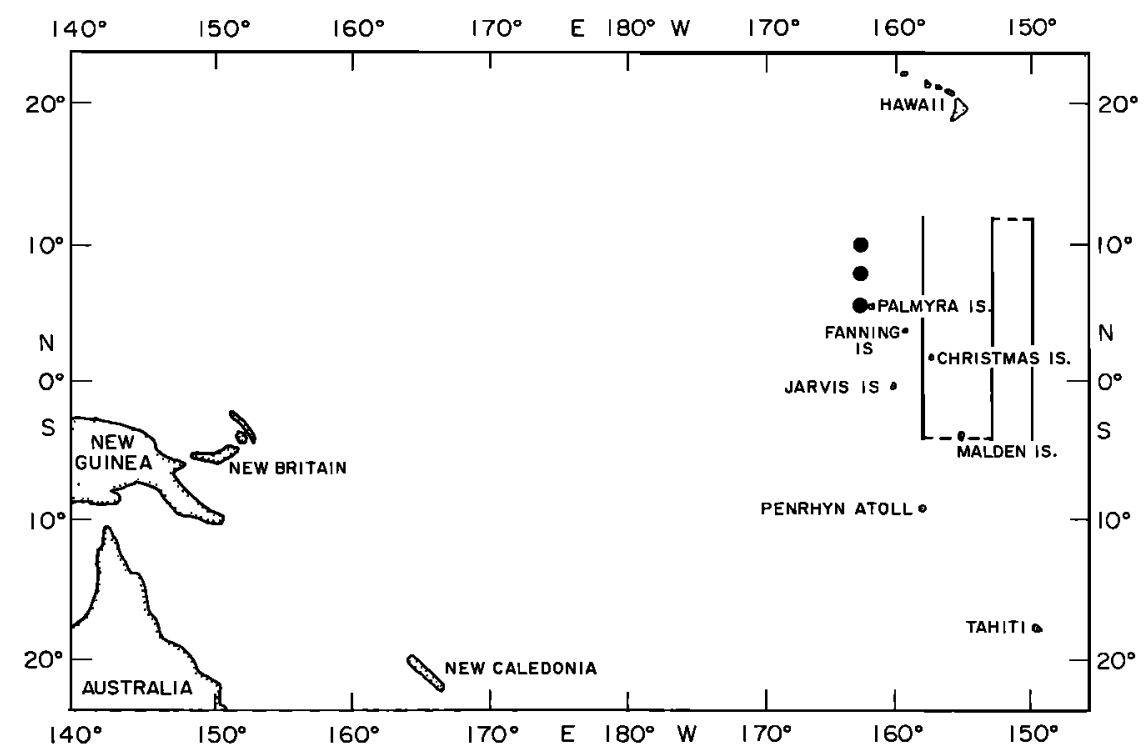

Fig. 1. Central Pacific Ocean showing the location of the Line Islands array, IESs (solid circles) along $162.5^{\circ} \mathrm{W}$. IESs at $6^{\circ}$ and $8^{\circ} \mathrm{N}$ were equipped with deep bottom pressure gauges. A shallow subsurface pressure gauge was located at Palmyra Island. Also shown is the NORPAX Hawaii-to-Tahiti Shuttle cruise track. CTD casts were made every degree between $4^{\circ} \mathrm{S}$ and $12^{\circ} \mathrm{N}$ along $150^{\circ}, 153^{\circ}$, and $158^{\circ} \mathrm{W}$; the casts used in this study were those taken near $6^{\circ} \mathrm{N}$.

thus suggest that travel time may be a good linear indicator of dynamic height. Since $c$ and $\rho$ depend on temperature and salinity differently, however, the relationship between $\tau$ and $D$ depends on the $T-S$ properties of the water column. An IES calibration thus varies regionally and may depend on the process leading to the variability in $\tau$ and $D$ (e.g., variability limited to the upper water column may need a different calibration from that extending throughout the entire column).

\section{CTD-Derived Calibration}

The CTD casts used in the present study were taken as part of the NORPAX Hawaii-to-Tahiti Shuttle Experiment [Wyrtki et al., 1981; Wyrtki and Kilonsky, 1984]; sites were spaced $1^{\circ}$ apart along three meridional legs $\left(150^{\circ}, 153^{\circ}\right.$, and $158^{\circ} \mathrm{W}$ ) between Hawaii and Tahiti (Figure 1). Casts were taken at each site approximately once a month between February 1979 and June 1980 , usually to 1000 dbar.

The plot of dynamic height anomaly relative to $1000 \mathrm{dbar}$, $\Delta D_{0 / 1000}$, as a function of synthesized travel time to the same level, $\tau_{0 / 1000}$, for all NORPAX CTD casts taken between $5.5^{\circ} \mathrm{N}$ and $6.5^{\circ} \mathrm{N}$ (Figure 2) shows the typical linear relationship $\left(r^{2}=0.94\right)$ found in these regressions. The slope, $m$, of -82.1 dyn m s${ }^{-1}$ is thus the "calibration" between $\Delta D_{0 / 1000}$ and $\tau_{0 / 1000}$.

The number of deep CTD casts near $6^{\circ} \mathrm{N}, 162^{\circ} \mathrm{W}$ (being limited, at present, to those made at the IES sites during this project) is insufficient to provide a full-water column calibration, and the extension of the 1000-dbar calibration to the bottom $(\sim 4300 \mathrm{~m})$ cannot be made without some prior knowledge of how $m$ varies as the lower reference level deepens. Because CTD casts in the tropics rarely extend beyond $1000 \mathrm{dbar}$, this knowledge is limited. However $\mathrm{L}$. Miller (personal communication, 1986), in a study of thermistor and CTD data collected near the equator at $110^{\circ} \mathrm{W}$, finds that the magnitude of $m$ drops by about $20 \%$ as the lower reference level deepens from 1000 dbar to below 3500 dbar (Figure 3). Thus if the same is true here, the full-water column calibration (i.e., $\Delta D_{0 / 4300}$ versus $\tau_{0 / 4300}$ ) should be about -66 dyn $\mathrm{m} \mathrm{s}^{-1}$.

\section{Relating Travel Time to Sea Surface Elevation and Dynamic Height}

Figure 4 illustrates the model used here. On the basis of the discussion in section 2, we make the initial assumption that variations in travel time $\tau_{\text {ref }}$, above some reference pressure level $p_{\text {ref }}$ close to the seafloor, are linearly proportional to variations in the dynamic height anomaly $\Delta D$ of the sea surface relative to that deep level. We also assume that the calibration may be frequency dependent and approach the problem in the frequency domain. Thus at some frequency $f>0$,

$$
\tilde{\tau}_{\mathrm{rer}}(f)=\frac{1}{m(f)} \Delta \bar{D}(f)
$$

where $m$ is called the calibration slope for consistency with Chiswell et al. [1986], and the tilde denotes the Fourier transform.

Since $p_{\text {ref }}$ is close to the seafloor, the average density $\rho_{b}$, and

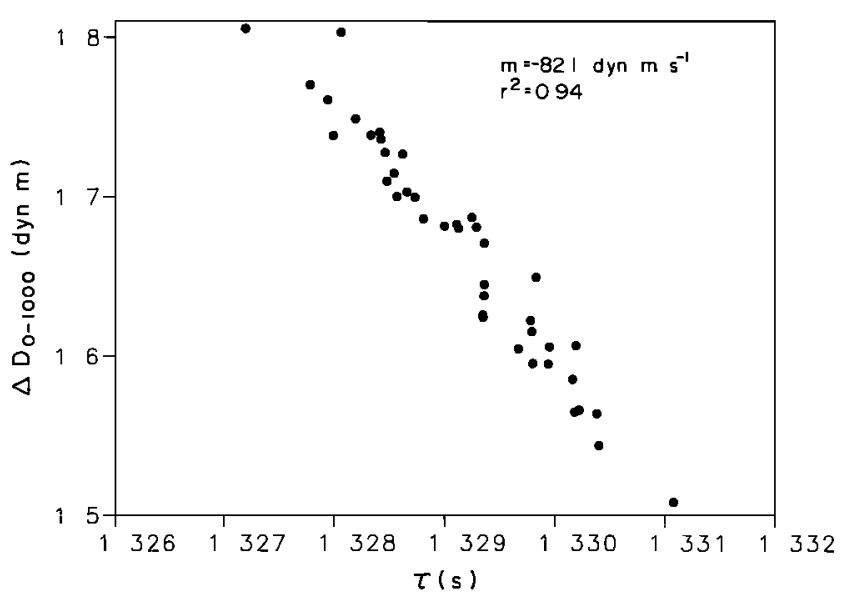

Fig. 2. Dynamic height anomaly relative to $1000 \mathrm{dbar}, \Delta D_{0 / 1000}$, as a function of synthesized travel time to the same level, $\tau_{0 / 1000}$, from all NORPAX CTD casts taken between $5.5^{\circ}$ and $6.5^{\circ} \mathrm{N}$. Slope $m$ and $r^{2}$ from the linear regression are also given. 


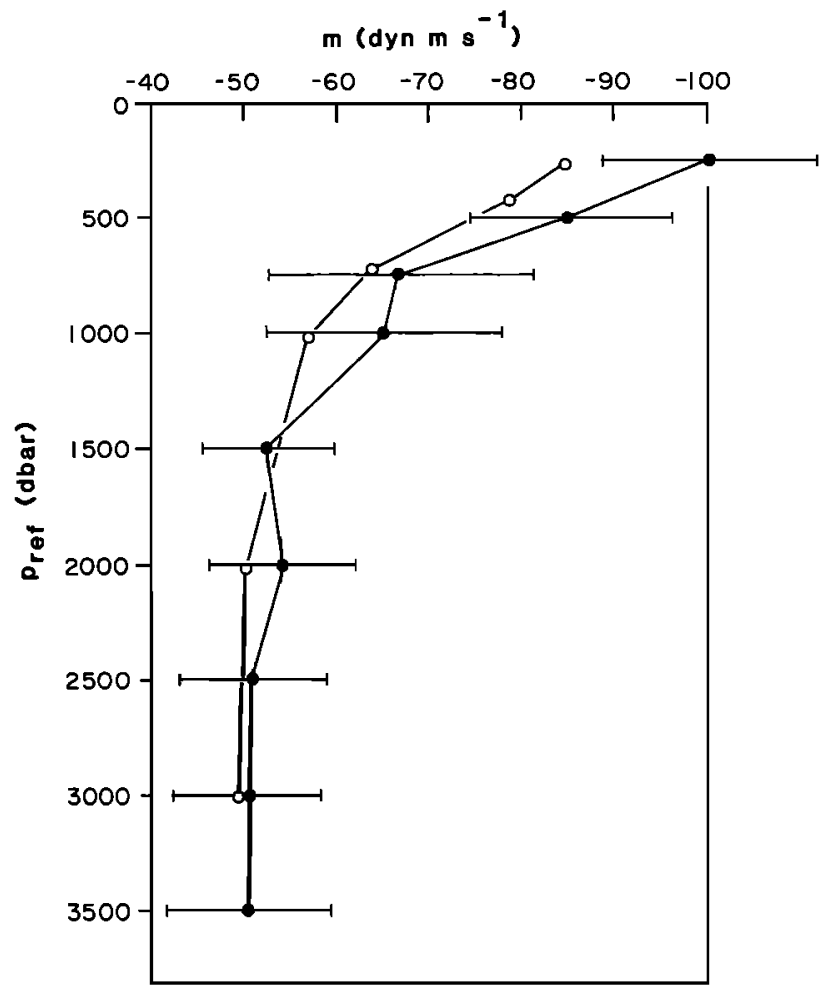

Fig. 3. IES calibration slope $m\left(\Delta D_{0 / \text { prer }}\right.$ versus $\left.\tau_{0 / \text { pref }}\right)$, as a function of the lower reference pressure level, $p_{\text {ref }}$, for the equator at $110^{\circ} \mathrm{W}$; solid circles are from CTD casts; open circles show combined thermistor data from D. Halpern and R. Weisberg, with $T-S$ curve derived from Mangum and Hayes [1983] (from L. Miller, personal communication, 1986).

the average speed of sound, $c_{b}$, below $p_{\text {ref }}$ are assumed constant in time. The total travel time to the bottom, $\tau$, is then

$$
\tilde{\tau}(f)=\frac{1}{m(f)} \Delta \tilde{D}(f)+\frac{2}{c_{b}} \tilde{H}_{2}(f)
$$

where $\mathrm{H}_{2}$ is the geometric distance between the seafloor and the reference pressure level. $\mathrm{H}_{2}$ is constant only if the ocean is baroclinically compensated; in general it varies according to

$$
H_{2}(t)=\eta(t)+H_{3}-H_{1}(t)
$$

where $\eta$ is sea level, $H_{1}$ is the geometric distance between the reference pressure level and the sea surface, and $H_{3}$ is the mean depth. Since $\mathrm{H}_{3}$ is a constant, the Fourier transform of (3) is

$$
\tilde{H}_{2}(f)=\tilde{\eta}(f)-\tilde{H}_{1}(f)
$$

From the definition of dynamic height [Neumann and Pierson, 1966], $H_{1}$ is given by

$$
H_{1}=\frac{10}{g} D=\frac{10}{g}\left(\Delta D+D_{\text {sld }}\right)
$$

where $g$ is the acceleration due to gravity, $\Delta D$ is the dynamic height anomaly and $D_{\text {std }}$ is the standard-ocean $\left(0^{\circ}, 35 \%\right)$ dynamic height. Because $D_{\text {std }}$ is constant,

$$
\tilde{H}_{1}(f)=\frac{10}{g} \Delta \tilde{D}(f)
$$

Substituting (4) and (5) into (2) yields

$$
\tilde{\tau}(f)=\left(\frac{1}{m(f)}-\frac{20}{g c_{b}}\right) \Delta \tilde{D}(f)+\frac{2}{c_{b}} \tilde{\eta}(f)
$$

A similar equation can be obtained for pressure. The pressure at the bottom, $p$, is (in decibars)

$$
p=p_{\text {ref }}+10^{-4} \rho_{b} g H_{2}
$$

so that

$$
\tilde{p}(f)=10^{-4} \rho_{b} g \tilde{H}_{2}(f)
$$

Substituting (4) and (5) into (7) gives

$$
\tilde{p}(f)=10^{-4} \rho_{b} g \tilde{\eta}(f)-10^{-3} \rho_{b} \Delta \tilde{D}(f)
$$

Equations (8) and (6) can be recast as

$$
\begin{gathered}
\alpha \tilde{p}(f)=\tilde{\eta}(f)-\beta \Delta \tilde{D}(f) \\
\tilde{\tau}(f)=\gamma \tilde{\eta}(f)-\delta(f) \Delta \tilde{D}(f)
\end{gathered}
$$

Equations (9) can be rearranged to express the dynamic height field in terms of two linear combinations of $\tau, \eta$, and $p$ :

$$
\begin{aligned}
& \Delta \tilde{D}(f)=[\tilde{\eta}(f)-\alpha \tilde{p}(f)] / \beta \\
& \delta(f) \Delta \tilde{D}(f)=\gamma \tilde{\eta}(f)-\tilde{\tau}(f)
\end{aligned}
$$

where

$$
\alpha=\frac{10^{4}}{\rho_{b} g} \quad \beta=\frac{10}{g} \quad \gamma=\frac{2}{c_{b}} \quad \delta(f)=-\left(\frac{1}{m(f)}-\frac{20}{g c_{b}}\right)
$$

Equations (10) can be regarded analogous to a linear response system [Jenkins and Watts, 1968] with $\gamma \eta-\tau$ as the response to $\Delta D$ or $(\eta-\alpha p) / \beta)$ with a transfer function $\delta(f)$, so that in the time domain, $\gamma \eta-\tau$ is the convolution of $\Delta D$ with the inverse Fourier transform of $\delta$.

This model has implicitly assumed negligible atmospheric pressure effects. Appendix A presents the modifications required to incorporate atmospheric pressure fluctuations in the model, for regions where this is necessary.

\section{INTERCOMPARISON}

Since atmospheric pressure was not available for Palmyra Island, sea level was computed from the measured shallow pressure, assuming that atmospheric pressure fluctuations were relatively small. This assumption was confirmed by at-

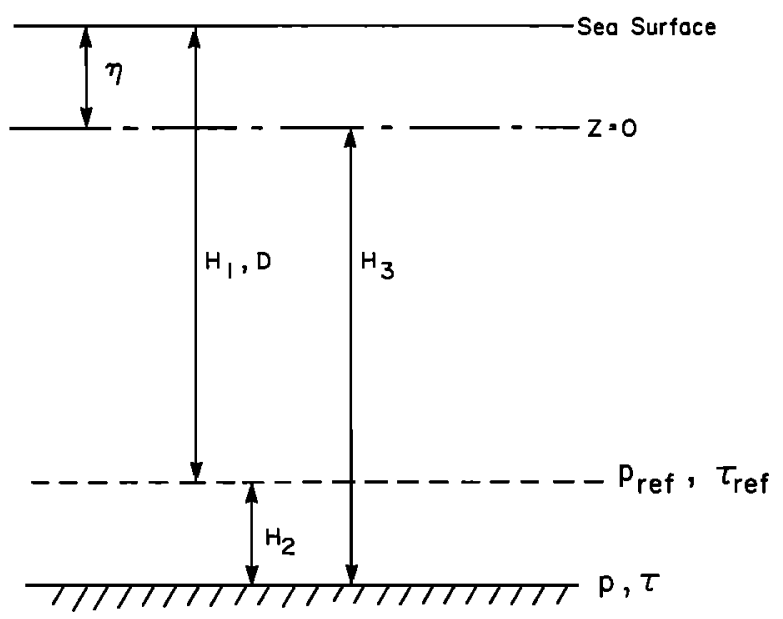

Fig. 4. Schematic diagram of relationships between dynamic height, travel times, sea level, and pressures (see text). 


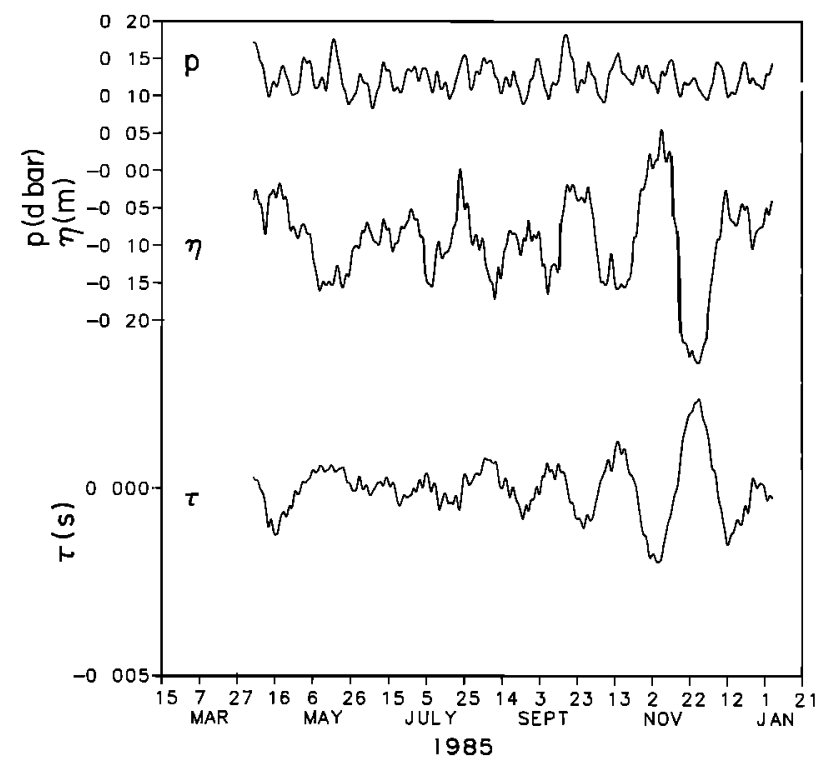

Fig. 5. Band-passed ( $-40-\mathrm{dB}$ points at 36 hours and 856 days) time series of travel time $\tau$, sea level $\eta$, and bottom pressure $p$, taken from the $6^{\circ} \mathrm{N}$ IES and Palmyra Island sites.

mospheric pressure measurements at Christmas Island (see Appendix A).

The time series of $\tau, \eta$, and $p$ and of the resulting series $(\eta-\alpha p) / \beta$ and $\gamma \eta-\tau$ are shown in Figures 5 and 6 (data analysis details are given in Appendix B). A failure of the Paroscientific Digiquartz sensor in the Palmyra Island pressure gauge caused a gradual long-term drift in the sea level record. For this reason, the time series are shown as bandpassed time series (upper and lower $-40 \mathrm{~dB}$ points at 36 hours and 856 days, respectively). The records of $(\eta-\alpha p) / \beta$ and $\gamma \eta-\tau$ (Figure 6) are visually well correlated, with the dominant 35 to 40 -day waves appearing quite similar.

To compare the contributions of each term in $\gamma \eta-\tau$ and $(\eta-\alpha p) / \beta$, we have plotted, in Figure 7 , the spectra of $\tau / \gamma, \eta$, and $\alpha p$. The spectra show that $\gamma \eta$ contributes only about $1 \%$ of the variance of $\gamma \eta-\tau$ and that the deep pressure $p$ influences $(\eta-\alpha p) / \beta$ strongly only at 2 -week period (principally $M f$ tide, which here appears broadened by the spectral analysis), and (to a lesser degree) at 5 -day period. Otherwise, $p$ generally contributes about $10 \%$ to the variance of $(\eta-\alpha p) / \beta$.

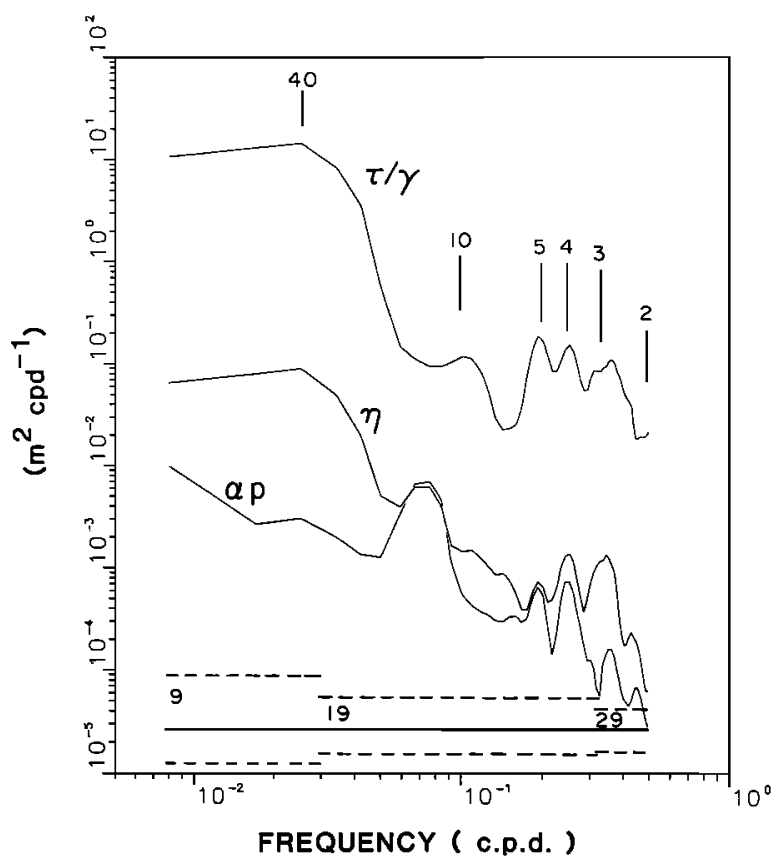

Fig. 7. Spectra of $\tau / \gamma, \eta$, and $\alpha p$. Vertical guidelines are drawn at indicated periods (in days) for comparison with other figures. The $95 \%$ confidence limits are drawn for the number of degrees of freedom as indicated.

The spectra of $\gamma \eta-\tau$ and $(\eta-\alpha p) / \beta$, shown in Figure 8, illustrate the elimination of the barotropic signal in (10), by the absence of $M f$ tidal peaks at 13.7 days despite their strong presence in the $p$ and $\eta$ records (Figure 7). The two spectra in Figure 8 are very similar: both show peaks with magnitudes comparable to the $95 \%$ confidence interval at $40,10,5,4$, and 3 days, indicating strong baroclinic signals at these periods.

The coherence squared between $\gamma \eta-\tau$ and $(\eta-\alpha p) / \beta$ (Figure 9) is almost always greater than the $95 \%$ significance level and exceeds the $99.9 \%$ significance value in bands coinciding with spectral peaks. However, it is not 1.0 , indicating that some of the variance is uncorrelated between the two variables. Apart from inadequacies in the assumption of a linear dependence of $\tau$ on $\Delta D$, this could be caused by the $70-\mathrm{km}$ separation of the IES and shallow pressure gauge (i.e., this "noise" is attributable to short-wavelength processes and island effects).

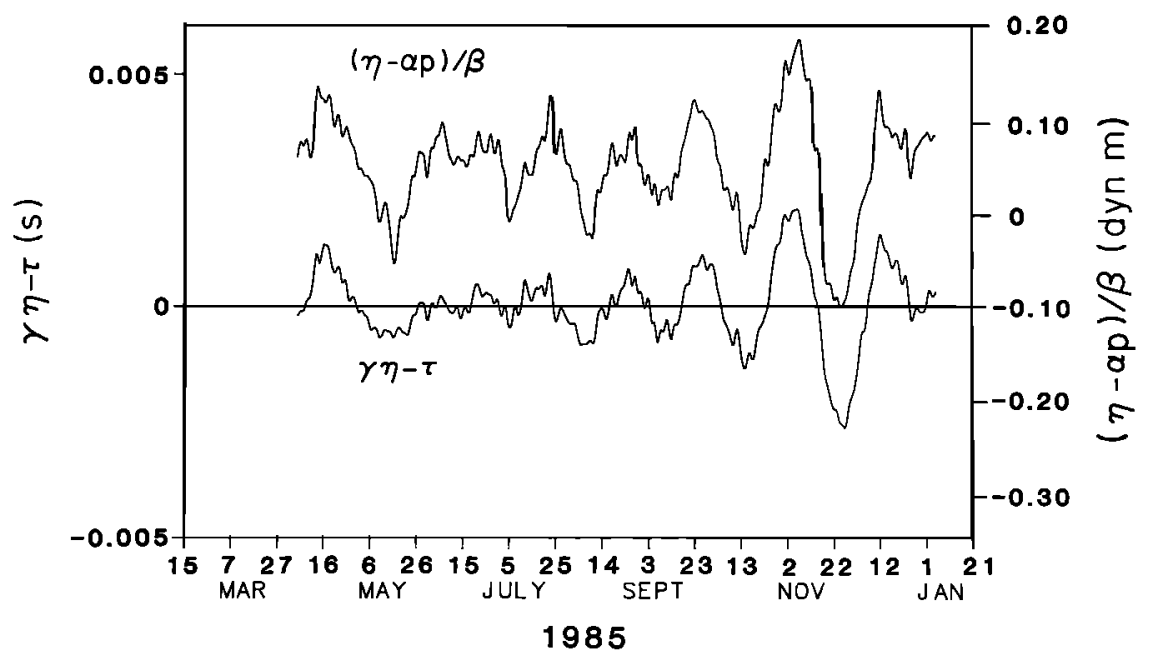

Fig. 6. Band-passed ( $-40-\mathrm{dB}$ points at 36 hours and 856 days) time series of $(\eta-\alpha p) / \beta$ and $\gamma \eta-\tau$. 


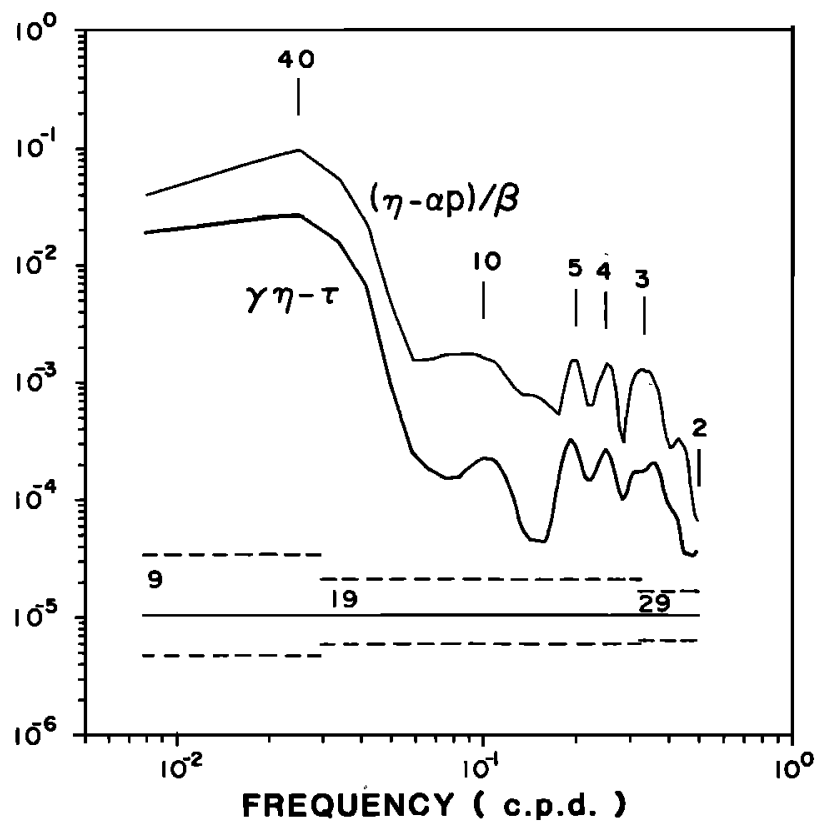

Fig. 8. Spectra of $(\eta-\alpha p) / \beta$ and $\gamma \eta-\tau$. Spectral units are (dyn $\mathrm{m})^{2} \mathrm{cpd}^{-1}$ and $\mathrm{s}^{2} \mathrm{cpd}^{-1}$, respectively; the spectrum of $\gamma \eta-\tau$ has been raised by 3 decades to facilitate comparison of the two spectra. Vertical guidelines and $95 \%$ confidence limits are as in Figure 7.

Since $\eta$ was measured in an island lagoon $70 \mathrm{~km}$ from the deep-sea measurements of $\tau$ and $p$, neither $\gamma \eta-\tau$ nor $(\eta-\alpha p) / \beta$ will precisely represent the true values at the IES site. Consequently, both $(\eta-\alpha p) / \beta$ and $\gamma \eta-\tau$ have some noise associated with them, and our analysis is that of a linear response system with noisy measurements of both "input" and "output" signals (see Appendix B).

The calibration slope $m$, computed from $\delta$, is plotted in Figure 10 at frequencies corresponding to peaks in the coherence squared. The indicated limits on $m$ are computed from Monte Carlo simulations of the expected fifth and ninety-fifth percentile values of the distribution of $\delta$.

At periods of near 40 days, $m$ is $-65 \pm 9$ dyn $\mathrm{m} \mathrm{s}^{-1}$, in excellent agreement with the depth-extended CTD-derived calibration of -66 dyn $\mathrm{m} \mathrm{s}^{-1}$ (section 2). These 40-day oscillations are seen in Figure 5 to have the largest amplitude, with about the same peak-to-peak range in dynamic height as the range in the CTD data $(\sim 0.3$ dyn $\mathrm{m}$, Figure 2$)$. This suggests

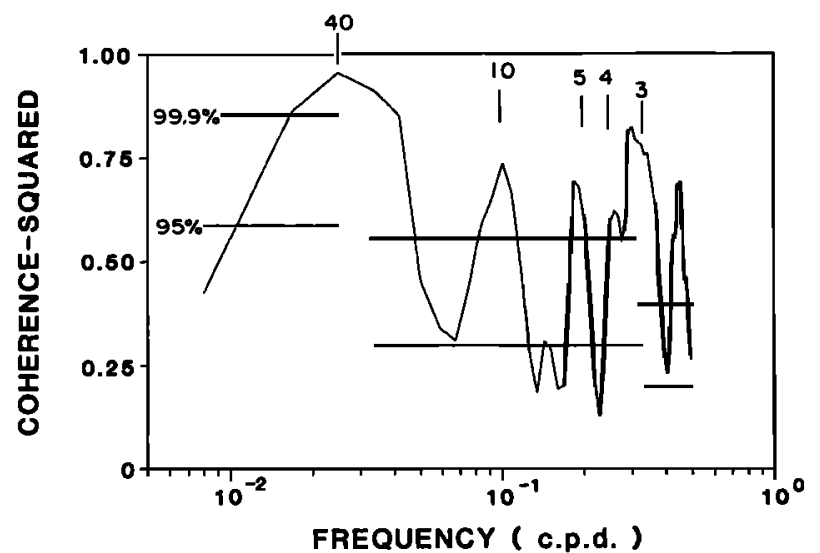

Fig. 9. Coherence squared between $(\eta-\alpha p) / \beta$ and $\gamma \eta-\tau$. Vertical guidelines are as in Figure 7; horizontal lines are levels of 95 and $99.9 \%$ significance.

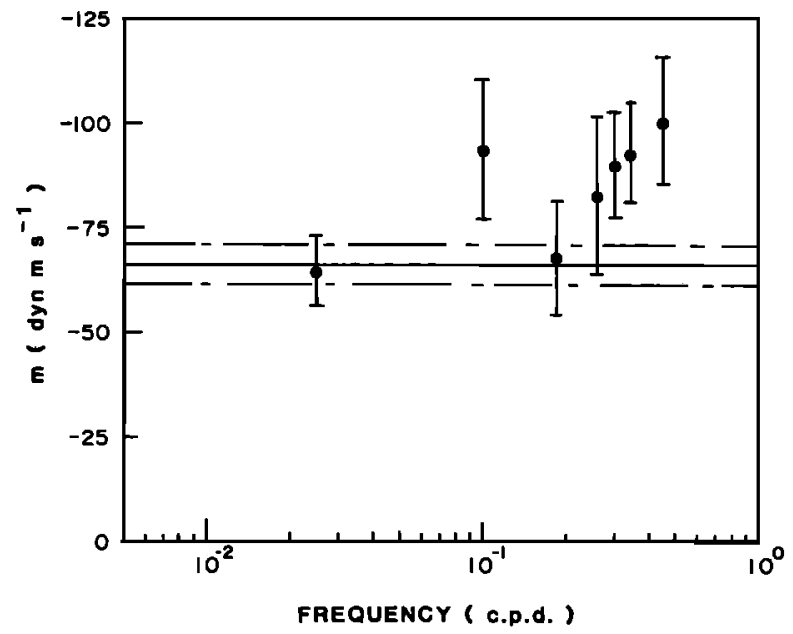

Fig. 10. IES calibration slope $m$, derived from equation $(10)$ at periods where peaks occur in the coherence squared. Error bars are expected fifth and ninety-fifth percentile values based on Monte Carlo simulations of a linear response system with noise at both input and output. The solid horizontal line is the depth-extended CTD-derived calibration slope, $-66 \mathrm{dyn} \mathrm{m} \mathrm{s}^{-1}$; dashed lines are 2 standard deviations from this estimate.

that the predominant contribution to the CTD-derived calibration may well have been similar 40-day variability, and this would explain the good agreement at low frequency between the two methods.

There is some evidence that $m$ changes with frequency. The general trend is for the magnitude of $m$ to increase with increasing frequency. At periods of 10,3 , and 2 days, $m$ is significantly greater than the CTD-derived value, at most about $50 \%$ greater. The trend is not monotonic, however: at 4 - and 5-day periods, $m$ does not differ significantly from the CTDderived value.

This apparent frequency dependence of $m$ suggests that oscillations in different frequency bands do have different vertical structure. The 40-day period waves are presumably examples of the 20- to 80-day period-oscillations observed by Mitchum and Lukas [1987], who attribute them to baroclinic instabilities in the equatorial current system having synoptic spatial scale [Legeckis, 1977]. Our data agree with previous observations that these "synoptic" oscillations have largest amplitude in October and November [Lukas, 1982]. The 10-day waves are probably equatorially trapped Rossbygravity waves similar to those seen in the eastern equatorial Pacific by Chiswell et al. [1987], whereas the 5-, 4-, 3- and 2-day waves fall in the equatorially trapped inertia-gravity wave band and are probably different meridional modes of these waves.

\section{Summary AND Conclusions}

Until now, the justification for using inverted echo sounders to measure baroclinic processes has relied heavily on temporally discrete CTD or expendable bathythermograph observations, and the assumption that the resulting calibration is applicable at all frequencies. Here (for the first time) we make a direct comparison of dynamic height derived from travel time with that derived independently from sea surface elevation and bottom pressure.

We have presumed that the differences between $(\eta-\alpha p) / \beta$ and $\gamma \eta-\tau$ arise predominantly through inadequacies of the assumption that $\tau$ and $\Delta D$ are linearly related as in (1). But 
noise in the measurements of $\eta, \tau$, and $p$ (some of it from topographic and physical separation effects; see Appendix B) also contributes to these differences.

The variable $(\eta-\alpha p) / \beta$ removes the barotropic component from the sea surface signal and is a precise measure of dynamic height (to the extent that $\eta$ accurately represents the open ocean sea level), since its derivation requires only the assumption that the near-bottom density is constant in time; the coefficients $\alpha$ and $\beta$ depend only on $\rho_{b}$ and $g$ and are easily determined. The variable $\gamma \eta-\tau$ incorporates a correction for sea level changes to the assumption that $\tau$ follows $\Delta D$, but this correction is small (the variance of $\eta$ is generally 2 decades lower than that of $\tau / \gamma)$. Thus $\gamma \eta-\tau$ and $(\eta-\alpha p) / \beta$ are practically independent measurements. Consequently, two factors confirm that $\gamma \eta-\tau$ can be used to measure $\Delta D$ : the very similar nature of the spectra of $\gamma \eta-\tau$ and $(\eta-\alpha p) / \beta$ (Figure 8) and the greater than $99.9 \%$ confidence in the coherence between the two variables at the spectral peaks (Figure 9).

It is clear that in certain bands, sea level is affected significantly by barotropic processes; these bands are not limited to those where one might a priori expect dominantly barotropic signals (e.g., at the $M f$ tidal period). A comparison of Figures 7 and 8 shows that near 5- and 4-day periods the spectrum of $\tau / \gamma$ is closer to the dynamic height spectrum (i.e., $(\eta-\alpha p) / \beta$ ) than is the spectrum of $\eta$. In these bands, then, even with the inherent uncertainty in IES calibration, acoustic travel time provides a better measure of dynamic height than does sea level.

This paper has presented a method for comparing dynamic height measured from acoustic travel time with that obtained from island sea level. In principle, varying mixtures of vertical modes could produce a frequency dependent IES calibration, $m(f)$, but this does not appear to have occurred to a significant extent during this deployment. At long periods, $m$ derived from the IES-pressure gauge comparison agrees well with the CTD-derived slope extended to $4000 \mathrm{~m}$ (though the applicability of the extension is otherwise uncertain). There is some evidence that $m$ depends on the processes leading to the variability, with higher-frequency dynamics having steeper calibration slopes; however, it appears that using the low-frequency value would underestimate the amplitude of these processes by at most $30 \%$.

\section{Appendix A: Inclusion of Atmospheric Pressure}

Atmospheric pressure was not measured at Palmyra Island. However, it was measured at Christmas (Kiritimati) Island $\left(2^{\circ} \mathrm{N}, 157^{\circ} 30^{\prime} \mathrm{W}\right)$, about $700 \mathrm{~km}$ to the southeast of Palmyra Island, coincident with the IES observations (except for the first month and for 25 days in August and September 1985 (P. Freitag, personal communication, 1986). Over large distances in the tropics, atmospheric pressure fluctuations are similar at periods of days [Brier and Simpson, 1969] to weeks [Madden and Julian, 1972], so that Christmas Island pressure may be used in the following order of magnitude estimate to show that ignoring atmospheric pressure has negligible effect for our deployment.

The bottom pressure $p_{b}$ is the sum of the hydrostatic pressure $p$ and atmospheric pressure $p_{a}$. Dropping the frequency dependence for simplicity,

$$
p_{b}=p+p_{a}
$$

Similarly, the measured shallow pressure $p_{s}$ includes atmospheric variations

$$
p_{s}=10^{-4} \rho_{s} g \eta+p_{a}
$$

or

$$
\eta=\frac{\left(p_{s}-p_{a}\right)}{10^{-4} \rho_{s} g}
$$

where $\rho_{s}$ is the density near the surface.

Equations (9) then become

$$
\begin{gathered}
\alpha p_{b}=\eta-\beta \Delta D+a p_{a} \\
\tau=\gamma \eta-\delta D
\end{gathered}
$$

Rearranging terms, substituting for $\eta$ from (A1), and writing $\varepsilon=\rho_{b} / \rho_{s}-1$, gives

$$
\begin{gathered}
\Delta D=\left[\frac{p_{s}}{10^{-4} \rho_{s} g}-\alpha\left(p_{b}+\varepsilon p_{a}\right)\right] / \beta \\
\delta \Delta D=\gamma \frac{\left(p_{s}-p_{a}\right)}{10^{-4} \rho_{s} g}-\tau
\end{gathered}
$$

Since atmospheric pressure was ignored earlier, $\eta$ in (10) is the same as $p_{s} /\left(10^{-4} \rho_{s} g\right)$ in (A2). The differences between (10) and (A2) are thus the replacement of $p_{b}$ by $\left(p_{b}+\varepsilon p_{a}\right)$ and the replacement of $p_{s}$ by $p_{s}-p_{a}$.

Christmas Island atmospheric pressure fluctuations had a peak-to-peak range of $0.07 \mathrm{dbar}$, compared with about 0.3 dbar in the shallow pressures and 0.1 dbar in the deep pressures at $6^{\circ} \mathrm{N}$ (see Figure 5). A CTD cast made at the IES deployment shows $\rho_{s}=1.022 \times 10^{3} \mathrm{~kg} \mathrm{~m}^{-3}$ and $\rho_{b}=1.044$ $\times 10^{3} \mathrm{~kg} \mathrm{~m}^{-3}$, so that $\varepsilon=0.022$. Thus, fluctuations in $\varepsilon p_{a}$ are about 2 orders of magnitude less than those of $p_{b}$, already small compared with $p_{s}$ (section 5). Similarly, since the amplitude of $\gamma \eta$ is an order of magnitude less than that of $\tau$ and since $p_{a}$ has only one-fifth the fluctuation amplitude of $p_{s}$, replacing $p_{s}$ with $p_{s-} p_{a}$ has little overall effect.

This argument demonstrates that the influence of $p_{a}$ on the total variance of dynamic height is negligible; however, at some individual frequency the effect could be significant. Consequently, spectra of $\Delta D$ and $\delta \Delta D$ were computed according to (A2) but were visually indistinguishable from the spectra computed according to (10), when plotted as in Figure 8.

\section{Appendix B: Data Analysis}

The $6^{\circ} \mathrm{N}$ data were first treated for the removal of tides by the response analysis method of Munk and Cartwright [1966]. The deep pressure record was corrected for the small temperature sensitivity of the pressure sensor and then detrended with an exponential trend in accordance with Watts and Kontoyiannis [1986]. The series were then band-pass filtered by subtracting very low pass filtered series from low-pass filtered series using a Gaussian filter. The resulting filter has its upper and lower $-40-\mathrm{dB}$ points at 36 hours and 856 days. At 5.5-, $4-, 3-$, and 1-day periods, the filter reduces by $-3,-6,-10$, and $-90 \mathrm{~dB}$, respectively. The lower $-3-\mathrm{dB}$ point is at 80 days; at 365-day periods the filter reduces by $-25 \mathrm{~dB}$.

Spectra were computed using the weighted overlappedsegment averaging method [Welch, 1967] combined with band averaging at the higher frequencies. This allows higher resolution at low frequencies and improved statistics at high frequencies. The spectra shown in Figures 7 and 8 are "recolored" to compensate for the effects of filtering. Expressions for the number of degrees of freedom and $95 \%$ confidence limits 
were obtained from Koopmans [1974], Welch [1967], and Thompson [1979].

Equations (10) can be regarded analogous to a linear response system [Jenkins and Watts, 1968] with $(\eta-\alpha p) / \beta$ as the input, $\gamma \eta-\tau$ as the output, and $\delta(f)$ as the transfer lunction. However, neither $\gamma \eta-\tau$ nor $(\eta-\alpha p) / \beta$ is physically a direct response to the other (the link is the assumption that $\tau$ and $\Delta D$ are linearly related). Our measurements of $\eta$ were made in an island lagoon $70 \mathrm{~km}$ from our measurements of $p$ and $\tau$. These measurements consequently contain noise associated with the separation and, in the case of $\eta$, noise from topographic effects (such as wind setup in the lagoon). Our analysis is thus that of a system having noise in the measured input $x$,

$$
x=(\eta-a p) / \beta+e_{x}
$$

and in the measured output $y$,

$$
y=\gamma \eta-\tau+e_{y}
$$

where $e_{x}$ and $e_{y}$ are uncorrelated with $(\eta-\alpha p) / \beta$ and $\gamma \eta-\tau$. The best estimate of the transfer function $\delta$ is then given by

$$
\delta=\sqrt{\left(S_{y} / S_{x}\right)}
$$

where $S_{x}$ and $S_{y}$ are the measured spectra of $x$ and $y$, respectively.

The limits on $m$ shown in Figure 10 were estimated from Monte Carlo simulations of the limits on $\delta$ computed for our processing. In these simulations a linear response system was set up with a known transfer function. Both input and output were contaminated with Gaussian white noise with the same signal-to-noise ratio, and the transfer function was computed according to equation $B 1$. These simulations show that equation B1 correctly computes $\delta$ and that the limits on $\delta$ depend on the amount of noise present. This noise was estimated from the observed coherence between $x$ and $y$ assuming equal signal-to-noise ratios for each variable.

Acknowledgments. This work is supported by the U. S. TOGA Project Office of NOAA through contract NA85AA-D-AC092, and by the National Science Foundation under grant OCE-8408640. G. Chaplin and $M$. Mulroney prepared, deployed, and recovered the IESs. $K$. Constantine was instrumental in all aspects of the field work. The efforts of the captains and crews of S/V Machias and R/V Kila were essential to the success of the cruises. K. Tracey and S. DeCarlo processed the IES and Palmyra data, respectively. D. R. Watts and J. T. Merill gave us helpful advice.

\section{REFERENCES}

Brier, G. W., and J. Simpson, Tropical cloudiness and rainfall related to pressure and tidal variations, Q. J. R. Meteorol. Soc., 95, 120$147,1969$.
Chap̄lin, G. F., and D. R. Watts, Inverted echo sounder development, Oceans ' $84,1,249-253,1984$.

Chiswell, S. M., D. R. Watts, and M. Wimbush, Using inverted echo sounders to measure dynamic height in the eastern equatorial $\mathbf{P a}$ cific during the 1982-83 El Niño, Deep Sea Res., 33, 981-991, 1986.

Chiswell, S. M., D. R. Watts, and M. Wimbush, Inverted echo sounder observations of variability in the eastern equatorial Pacific during the 1982-83 El Niño, Deep Sea Res., 34, 313-327, 1987.

Jenkins, G. M., and D. G. Watts, Spectral Analysis and its Applications, 525 pp., Holden-Day, San Francisco, 1968.

Koopmans, L. H., The Spectral Analysis of Time Series, 366 pp., Academic, Orlando, Fla., 1974.

Legeckis, R., Long waves in the eastern equatorial Pacific Ocean: A view from a geostationary satellite, Science, 197, 1179-1181, 1977.

Lukas, R., Annual and interannual modulation of 30-day waves in the central equatorial Pacific Ocean, Eos Trans. AGU, 63, 974, 1982.

Madden, R. A., and P. R. Julian, Description of global-scale circulation cells in the tropics with a 40-50 day period, J. Atmos. Sci., $29,1109-1123,1972$.

Mangum, L. J., and S. P. Hayes, CTD $/ \mathrm{O}_{2}$ measurements during 1980 and 1981 as part of the Equatorial Pacific Ocean Climate Study (EPOCS), NOAA Data Rep. ERL PMEL-9, 621 pp., Pac. Mar. Environ. Lab., Seattle, Wash., 1983.

Miller, L. L., D. R. Watts, and M. Wimbush, Oscillations of dynamic topography in the eastern equatorial Pacific, J. Phys. Oceanogr., 15 , 1759-1770, 1985.

Mitchum, G., and R. Lukas, The latitude-frequency structure of Pacific sea-level variance, J. Phys. Oceanogr., 17, 2362-2365, 1987.

Munk, W. H., and D. E. Cartwright, Tidal spectroscopy and prediction, Philos. Trans. R. Soc. London, Ser. A, 259, 533-581, 1966.

Neumann, G. H., and W. J. Pierson, Principles of Physical Oceanography, 545 pp., Prentice-Hall, Englewood Cliffs, N. J., 1966.

Thompson, R. O. R. Y., Coherence significance levels, J. Atmos. Sci., 36, 2020-2021, 1979.

Watts, D. R., and W. E. Johns, Gulf Stream meanders: Observations on propagation and growth, J. Geophys. Res., 87, 9467-9476, 1982.

Watts, D. R., and H. Kontoyiannis, Deep-ocean bottom pressure and temperature sensors report: Methods and data, Tech. Rep. 86-8, 111 pp., Grad. Sch. of Oceanogr., Univ. of R. I., Narragansett, 1986.

Watts, D. R., and H. T. Rossby, Measuring dynamic height with inverted echo sounders: Results from MODE, J. Phys. Oceanogr., 7, 345-358, 1977.

Welch, P. D., The use of fast Fourier transform for the estimation of power spectra: A method based on time averaging over short, modified periodograms, IEEE Trans. Audio Electroacoust., $A U-15$, 70-73, 1967.

Wyrtki, K., and B. Kilonsky, Mean water and current structure during the Hawaii-to-Tahiti shuttle experiment, J. Phys. Oceanogr., 14, 242-254, 1984.

Wyrtki, K., E. Firing, D. Halpern, R. Knox, G. J. McNally, W. C. Patzert, E. D. Stroup, B. A. Taft, and R. Williams, The Hawaii to Tahiti Shuttle experiment, Science, 211, 22-28, 1981.

S. M. Chiswell and R. Lukas, Joint Institute for Marine and Atmospheric Research, University of Hawaii at Manoa, 1000 Pope Road, Honolulu, HI 96822.

M. Wimbush, Graduate School of Oceanography, University of Rhode Island, Narragansett, RI 02882.

(Received October 21, 1987; accepted October 26, 1987.) 